\title{
NUMERICAL SOLUTION OF AN INVERSE INITIAL BOUNDARY VALUE PROBLEM FOR THE WAVE EQUATION IN THE PRESENCE OF CONDUCTIVITY IMPERFECTIONS OF SMALL VOLUME
}

\author{
Mark Asch ${ }^{1}$, Marion Darbas $^{1}$ And Jean-Baptiste Duval ${ }^{1}$
}

\begin{abstract}
We consider the numerical solution, in two- and three-dimensional bounded domains, of the inverse problem for identifying the location of small-volume, conductivity imperfections in a medium with homogeneous background. A dynamic approach, based on the wave equation, permits us to treat the important case of "limited-view" data. Our numerical algorithm is based on the coupling of a finite element solution of the wave equation, an exact controllability method and finally a Fourier inversion for localizing the centers of the imperfections. Numerical results, in 2- and 3-D, show the robustness and accuracy of the approach for retrieving randomly placed imperfections from both complete and partial boundary measurements.
\end{abstract}

Mathematics Subject Classification. 35R30, 35L05, 65M60.

Received September 4, 2009. Revised April 26, 2010.

Published online August 6, 2010.

\section{INTRODUCTION}

The localization of small imperfections is of great importance since there are numerous practical applications, particularly in the fields of medical imaging and nondestructive testing of materials. Generally, when we seek to localize an imperfection contained in a bounded domain, we need to solve an inverse problem for retrieving the geometry of the imperfection.

The determination of conductivity profiles from knowledge of boundary measurements has received a great deal of attention (see, for example, $[1,2,5,20,32]$ ). However, the reconstruction of imperfections within a dynamical (i.e. time-dependent) framework has not been widely investigated. The present paper is a first attempt to implement an effective numerical method to determine the location of small-sized, conductivity imperfections inside a homogeneous medium from dynamical measurements on a part of the boundary. In imaging, this is known as "limited-view data".

The inverse problem considered in this paper is more complicated from the mathematical point of view and more interesting from the point of view of applications than the one solved in [5,32] because one often cannot get measurements for all $t$ or on the whole boundary, and so one cannot, by taking a Fourier transform in the time variable, reduce our dynamic inverse problem to the Helmholtz equation considered in [5,32]. Previous numerical investigations have concentrated on time independent equations with full data - see for example, $[6,7,12]$.

Keywords and phrases. Wave equation, exact controllability, inverse problem, finite elements, Fourier inversion.

1 LAMFA-CNRS UMR 6140, Université de Picardie Jules Verne, 80039 Amiens, France. mark.asch@u-picardie.fr 


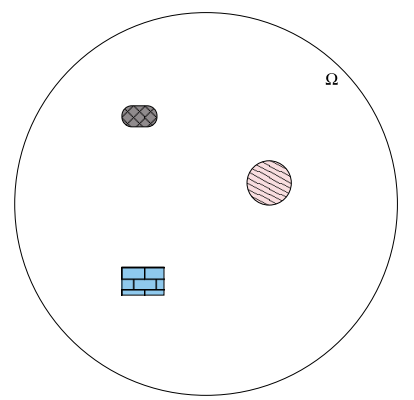

Figure 1. An example of a 2-D domain containing three imperfections, $z_{1}+\alpha B_{1}, z_{2}+\alpha B_{2}, z_{3}+\alpha B_{3}$.

This article is based on three sources: the Hilbert Uniqueness Method (HUM) formulated by Lions - see [30]; the numerical application of the above method to exact boundary control of the wave equation by Glowinski et al. - see [26]; the theoretical results of the problem of detection for the wave equation by Ammari - see [2].

This paper is structured as follows. We begin, in Section 2, by defining some notation and then formulate the inverse initial boundary value problem. We also address the identification procedure by the Fourier method. In Section 3, we recall the numerical algorithm for the Hilbert Uniqueness Method of Lions [30]. We present the numerical method used for the dynamic detection problem in Section 4. Then numerical results obtained from simulations are shown in Section 5. Finally, some conclusions and perspectives are reported in the last section.

\section{DyNAMiC LOCALIZATION THEORY}

In this part, we recall the different results contained in the article of Ammari (see [2] for the full details) on which the numerical algorithm and the subsequent simulations will be based.

\subsection{Some notation and presentation of the inverse problem}

\subsubsection{Some notation}

Let $\Omega$ be a bounded, smooth subdomain of $\mathbb{R}^{d}, d=2,3$, with for simplicity, a smooth boundary $\partial \Omega$, with $n$ denoting the outward unit normal to $\partial \Omega$. We suppose that $\Omega$ contains a finite number $m$ of imperfections, each of the form $z_{j}+\alpha B_{j}$, where $B_{j} \subset \mathbb{R}^{d}$ is a bounded, smooth domain containing the origin (see Fig. 1). This gives a collection of imperfections of the form $B_{\alpha}=\cup_{j=1}^{m}\left(z_{j}+\alpha B_{j}\right)$. The points $z_{j} \in \Omega, j=1, \ldots, m$, that define the locations of the imperfections are assumed to satisfy two distance conditions:

$$
\left\{\begin{array}{l}
\left|z_{j}-z_{l}\right| \geq d_{0}>0 \forall j \neq l \\
\operatorname{dist}\left(z_{j}, \partial \Omega\right) \geq d_{0}>0 \forall j
\end{array}\right.
$$

We also assume that $\alpha>0$, the common order of magnitude of the diameters of the imperfections, is sufficiently small so that these are disjoint and their distance to $\mathbb{R}^{d} \backslash \bar{\Omega}$ is larger than $d_{0} / 2$.

\subsubsection{Presentation of the inverse problem}

Let $\gamma_{0}$ denote the conductivity of the background medium which, for simplicity, we shall assume is constant. Let $\gamma_{j}$ be the constant conductivity of the $j$-th imperfection, $z_{j}+\alpha B_{j}$. We define the piecewise constant conductivity

$$
\gamma_{\alpha}(x)= \begin{cases}\gamma_{0}, & \text { if } x \in \Omega \backslash \bar{B}_{\alpha}, \\ \gamma_{j}, & \text { if } x \in z_{j}+\alpha B_{j} .\end{cases}
$$


Consider the initial boundary value problem for the wave equation, in the presence of the imperfections,

$$
\begin{cases}\frac{\partial^{2} u_{\alpha}}{\partial t^{2}}-\nabla \cdot\left(\gamma_{\alpha} \nabla u_{\alpha}\right)=0 & \text { in } \Omega \times(0, T), \\ u_{\alpha}=f & \text { on } \partial \Omega \times(0, T), \\ \left.u_{\alpha}\right|_{t=0}=u^{0},\left.\frac{\partial u_{\alpha}}{\partial t}\right|_{t=0}=u^{1} & \text { in } \Omega .\end{cases}
$$

Define $u$ to be the solution of the wave equation in the absence of any imperfections, satisfying

$$
\begin{cases}\frac{\partial^{2} u}{\partial t^{2}}-\nabla \cdot\left(\gamma_{0} \nabla u\right)=0 & \text { in } \Omega \times(0, T), \\ u=f & \text { on } \partial \Omega \times(0, T), \\ \left.u\right|_{t=0}=u^{0},\left.\frac{\partial u}{\partial t}\right|_{t=0}=u^{1} & \text { in } \Omega .\end{cases}
$$

Here $T>0$ is a final observation time, and the initial conditions $\varphi, \psi \in C^{\infty}(\bar{\Omega})$ and the boundary condition $f \in C^{\infty}\left(0, T ; C^{\infty}(\partial \Omega)\right)$ are subject to the compatibility conditions at the initial time,

$$
\left.\partial_{t}^{2 l} f\right|_{t=0}=\left.\left(\gamma_{0}\right)^{l}\left(\Delta^{l} u^{0}\right)\right|_{\partial \Omega} \text { and }\left.\partial_{t}^{2 l+1} f\right|_{t=0}=\left.\left(\gamma_{0}\right)^{l}\left(\Delta^{l} u^{1}\right)\right|_{\partial \Omega}, \quad l=1,2, \ldots
$$

These ensure that the initial boundary value problem $(2.3)$ has a unique solution in $C^{\infty}([0, T] \times \bar{\Omega})$; see $[23]$. The transmission problem for the wave equation (2.2) has a unique weak solution $u_{\alpha} \in C^{0}\left(0, T ; H^{1}(\Omega)\right) \cap$ $C^{1}\left(0, T ; L^{2}(\Omega)\right)$; see, for example, [30]. Moreover, it can be shown that $\left.\frac{\partial u_{\alpha}}{\partial n}\right|_{\partial \Omega}$ belongs to $L^{2}\left(0, T ; L^{2}(\partial \Omega)\right)$.

We employ standard $L^{2}$-based Sobolev spaces to measure function regularity. The notation $H^{s}$ denotes those functions which, along with all their derivatives of order less than or equal to $s$, are in $L^{2}$. In particular, $H_{0}^{1}$ denotes the closure of $C_{0}^{\infty}$ in the norm $H^{1}$. Sobolev spaces with negative indices are defined by duality, using an $L^{2}$-inner product. We shall only need one such space, namely, $H^{-1}$, which is defined as the dual of $H_{0}^{1}$.

The inverse problem is: determine the centers of the imperfections from the knowledge of boundary data, generated numerically,

$$
\frac{\partial u_{\alpha}}{\partial n} \text { on } \Gamma_{c} \times(0, T)
$$

where $\Gamma_{c} \subseteq \partial \Omega$ is a part of the boundary that must satisfy certain geometrical constraints, and $(0, T)$ is the finite time-interval during which the measurements are recorded. It is also possible to compute certain properties of the shapes of the imperfections $B_{\alpha}$, though this is not treated here. For this purpose, Ammari, in [2], has developed an asymptotic method based on appropriate averaging of dynamic boundary measurements, using particular background solutions as weights. These particular solutions are constructed, when measurements are available on all or a part of the boundary of the domain, by the control method of Section 3 .

The first fundamental step in the design of the reconstruction method is the derivation of an asymptotic formula for $\left.\frac{\partial u_{\alpha}}{\partial \nu_{j}}\right|_{\partial\left(z_{j}+\alpha B_{j}\right)}+$ on the outer boundaries of the imperfections, in terms of the reference solution $u$, the location $z_{j}$ of the imperfection $z_{j}+\alpha B_{j}$, and the geometry of $B_{j}$. The next step is the use of this asymptotic formula to derive integral boundary formulae with a judicious choice of test functions and is based on the geometrical control method and the solution of Volterra-type integral equations. We will show that these boundary integral formulae form the basis of very effective numerical identification algorithms, as was predicted in [2]. We note that an analogous approach may be applied to the full (time-dependent) Maxwell equations with small imperfections of different electric permittivity or magnetic permeability (or both) - see [12] for the time-harmonic case.

\subsection{The asymptotic formula and the identification procedure}

Following [2], we let $\beta \in C_{0}^{\infty}(\Omega)$ be a cutoff function such that $\beta(x) \equiv 1$ in a subdomain $\Omega^{\prime}$ of $\Omega$ that contains the imperfections $B_{\alpha}$. For an arbitrary $\eta \in \mathbb{R}^{d}$, we assume that we are in possession of the boundary 
measurements of

for

$$
\frac{\partial u_{\alpha}}{\partial n} \text { on } \Gamma_{c} \times(0, T)
$$

$$
u^{0}(x)=\mathrm{e}^{\mathrm{i} \eta \cdot x}, u^{1}(x)=-i \sqrt{\gamma_{0}}|\eta| \mathrm{e}^{\mathrm{i} \eta \cdot x} \text { and } f(x, t)=\mathrm{e}^{\mathrm{i} \eta \cdot x-i \sqrt{\gamma_{0}}|\eta| t} .
$$

This particular choice of plane wave data $u^{0}, u^{1}$ and $f$ implies that the background solution $u$ of the wave equation (2.3) is given explicitly by

$$
u(x, t)=u_{\eta}(x, t)=\mathrm{e}^{\mathrm{i} \eta \cdot x-i \sqrt{\gamma_{0}}|\eta| t} \text { in } \Omega \times(0, T) .
$$

Suppose that $T$ and the part $\Gamma_{c}$ of the boundary $\partial \Omega$ are such that they geometrically control $\Omega$, which roughly means that every geometrical optics ray, starting at any point $x \in \Omega$ at time $t=0$, hits $\Gamma_{c}$ before time $T$ at a non-diffractive point - see [11,15]. Then from [30], it follows that for any $\eta \in \mathbb{R}^{d}$, we can construct by the Hilbert uniqueness method (HUM), a unique $g_{\eta} \in H_{0}^{1}\left(0, T ; L^{2}(\Gamma)\right)$ in such a way that the unique weak solution $w_{\eta}$ in $C^{0}\left(0, T ; L^{2}(\Omega)\right) \cap C^{1}\left(0, T ; H^{-1}(\Omega)\right)$ of the wave equation

$$
\begin{cases}\frac{\partial^{2} w_{\eta}}{\partial t^{2}}-\nabla \cdot\left(\gamma_{0} \nabla w_{\eta}\right)=0 & \text { in } \Omega \times(0, T), \\ w_{\eta}=g_{\eta} & \text { on } \Gamma_{c} \times(0, T), \\ w_{\eta}=0 & \text { on } \partial \Omega \backslash \bar{\Gamma}_{c} \times(0, T), \\ \left.w_{\eta}\right|_{t=0}=\beta(x) \mathrm{e}^{\mathrm{i} \eta \cdot x} & \in H_{0}^{1}(\Omega), \\ \left.\frac{\partial w_{\eta}}{\partial t}\right|_{t=0}=0 & \text { in } \Omega,\end{cases}
$$

satisfies $w_{\eta}(T)=\partial_{t} w_{\eta}(T)=0$.

Next, for any $\eta \in \mathbb{R}^{d}$, let $\theta_{\eta}$ be the solution to the Volterra equation of the second kind:

$$
\left\{\begin{array}{l}
\frac{\partial \theta_{\eta}(x, t)}{\partial t}+\int_{t}^{T} \mathrm{e}^{-\mathrm{i} \sqrt{\gamma_{0}}|\eta|(s-t)}\left(\theta_{\eta}(x, s)-\mathrm{i} \sqrt{\gamma_{0}}|\eta| \frac{\partial \theta_{\eta}(x, t)}{\partial t}\right) \mathrm{d} s \\
=g_{\eta}(x, t) \text { for } x \in \Gamma_{c}, t \in(0, T), \\
\theta_{\eta}(x, 0)=0 \text { for } x \in \Gamma_{c} .
\end{array}\right.
$$

The existence and uniqueness of this $\theta_{\eta}$ in $H^{1}\left(0, T ; L^{2}\left(\Gamma_{c}\right)\right)$, for any $\eta \in \mathbb{R}^{d}$, can be established using the resolvent kernel. Since $g_{\eta} \in H_{0}^{1}\left(0, T ; L^{2}\left(\Gamma_{c}\right)\right)$, the solution $\theta_{\eta}$ belongs, in fact, to $H^{2}\left(0, T ; L^{2}\left(\Gamma_{c}\right)\right)$. We also note from differentiation of (2.7) with respect to $t$ that $\theta_{\eta}$ is the unique solution of the ODE

$$
\begin{cases}\partial_{t} \theta_{\eta}-\theta_{\eta}=\mathrm{e}^{\mathrm{i} \sqrt{\gamma_{0}}|\eta| t} \partial_{t}\left(\mathrm{e}^{-\mathrm{i} \sqrt{\gamma_{0}}|\eta| t} g_{\eta}\right) & \text { for } x \in \Gamma_{c}, t \in(0, T) \\ \theta_{\eta}(x, 0)=\partial_{t} \theta_{\eta}(x, T)=0 & \text { for } x \in \Gamma_{c}\end{cases}
$$

It also immediately follows from this observation that $\theta_{\eta}$ belongs to $H^{2}\left(0, T ; L^{2}(\Gamma)\right)$ since $g_{\eta} \in H_{0}^{1}\left(0, T ; L^{2}(\Gamma)\right)$.

To identify the locations and certain properties of the small imperfections $B_{\alpha}$, we average the boundary measurements $\left.\frac{\partial u_{\alpha}}{\partial n}\right|_{\Gamma_{c} \times(0, T)}$, using the solution $\theta_{\eta}$ to the Volterra equation (2.7) or, equivalently, the ODE (2.8) as a function of $\eta$. The following result holds.

Theorem 2.1 (Ammari). Let $\eta \in \mathbb{R}^{d}, d=2$, 3. Let $u_{\alpha}$ be the unique solution in $C^{0}\left(0, T ; H^{1}(\Omega)\right) \cap C^{1}(0, T$; $L^{2}(\Omega)$ ) to the wave equation (2.2) with

$$
u^{0}(x)=u_{\eta}^{0}(x)=\mathrm{e}^{\mathrm{i} \eta \cdot x}, u^{1}(x)=u_{\eta}^{1}(x)=-\mathrm{i} \sqrt{\gamma_{0}}|\eta| \mathrm{e}^{\mathrm{i} \eta \cdot x}
$$

and

$$
f(x, t)=f_{\eta}(x, t)=\mathrm{e}^{\mathrm{i} \eta \cdot x-i \sqrt{\gamma_{0}}|\eta| t} .
$$


Suppose that $\Gamma_{c}$ and $T$ geometrically control $\Omega[15]$; then we have

$$
\begin{aligned}
\int_{0}^{T} \int_{\Gamma}\left[\theta_{\eta}\left(\frac{\partial u_{\alpha}}{\partial n}-\frac{\partial u}{\partial n}\right)+\partial_{t} \theta_{\eta} \partial_{t}\left(\frac{\partial u_{\alpha}}{\partial n}-\frac{\partial u}{\partial n}\right)\right] & =-\int_{0}^{T} \int_{\Gamma} \mathrm{e}^{\mathrm{i} \sqrt{\gamma_{0}}|\eta| t} \partial_{t}\left(\mathrm{e}^{-\mathrm{i} \sqrt{\gamma_{0}}|\eta| t} g_{\eta}\right)\left(\frac{\partial u_{\alpha}}{\partial n}-\frac{\partial u}{\partial n}\right) \\
& =\alpha^{d} \sum_{j=1}^{m}\left(\frac{\gamma_{0}}{\gamma_{j}}-1\right) \mathrm{e}^{2 \mathrm{i} \eta \cdot z_{j}}\left[M_{j}(\eta) \cdot \eta-|\eta|^{2}\left|B_{j}\right|\right]+o\left(\alpha^{d}\right),
\end{aligned}
$$

where $\theta_{\eta}$ is the unique solution to the $O D E(2.8)$, with $g_{\eta}$ defined as the boundary control in $(2.6)$, and $M_{j}$ is the polarization tensor of $B_{j}$, defined by

$$
\left(M_{j}\right)_{k, l}=e_{k} \cdot\left(\int_{\partial B_{j}}\left(\nu_{j}+\left(\frac{\gamma_{0}}{\gamma_{j}}-1\right) \frac{\partial \Phi_{j}}{\partial \nu_{j}}(y)\right) y \cdot e_{l} \mathrm{~d} s_{j}(y)\right)
$$

Here $\left(e_{1}, \ldots, e_{d}\right)$ is an orthonormal basis of $\mathbb{R}^{d}, \nu_{j}$ is the outward normal of the $j$-th imperfection and $\Phi_{j}$ is the solution of a Laplace equation in the $j$-th imperfection.

See [2] for the full formulation and details of the proof. This theorem is fundamental for our identification problems.

Remark 2.1. In this result, the control function is a technical device that is used in an adjoint formulation based on integration-by-parts. This control does not have a physical interpretation. Its role is to reduce the identification problem to a quantity that can be easily calculated on the boundary.

Remark 2.2. The choice of plane-wave initial data is only one of many possible choices. If we choose other initial functions, we indeed obtain different imaging algorithms (see [10]) but the complexity of the inverse problem remains the same, since we still have to compute the same boundary control.

We can now proceed to describe our identification procedure which is based on Theorem 2.1. We neglect the asymptotically small remainder in the asymptotic formula $(2.1)$ and define $\Lambda_{\alpha}(\eta)$ by

$$
\begin{aligned}
\Lambda_{\alpha}(\eta) & =-\int_{0}^{T} \int_{\Gamma} \mathrm{e}^{\mathrm{i} \sqrt{\gamma_{0}}|\eta| t} \partial_{t}\left(\mathrm{e}^{-\mathrm{i} \sqrt{\gamma_{0}}|\eta| t} g_{\eta}\right)\left(\frac{\partial u_{\alpha}}{\partial n}-\frac{\partial u}{\partial n}\right) \\
& \approx \alpha^{d} \sum_{j=1}^{m}\left(\frac{\gamma_{0}}{\gamma_{j}}-1\right) \mathrm{e}^{2 \mathrm{i} \eta \cdot z_{j}}\left[M_{j}(\eta) \cdot \eta-|\eta|^{2}\left|B_{j}\right|\right] .
\end{aligned}
$$

The function $\Lambda_{\alpha}(\eta)$ is computed in the following way. First, we construct the control $g_{\eta}$ in (2.6) for given $\eta \in \mathbb{R}^{d}$. Then from the boundary measurements $\left.\frac{\partial u_{\alpha}}{\partial n}\right|_{\Gamma \times(0, T)}$, and the explicit knowledge of $\frac{\partial u}{\partial n}$, we form the weighted boundary integrals that appear in the above expression of $\Lambda_{\alpha}(\eta)$.

Recall that the function $\mathrm{e}^{2 \mathrm{i} \eta \cdot z_{j}}$ is exactly the Fourier transform (up to a multiplicative constant) of the Dirac function $\delta_{-2 z_{j}}$ (a point mass located at $-2 z_{j}$ ). From Theorem 2.1, it follows that the function $\Lambda_{\alpha}(\eta)$ is (approximately) the Fourier transform of a linear combination of derivatives of point masses, or

$$
\breve{\Lambda}_{\alpha}(x) \approx \alpha^{d} \sum_{j=1}^{m} L_{j}\left(\delta_{-2 z_{j}}\right)(x)
$$

where $L_{j}$ is a second order, constant coefficient, differential operator whose coefficients depend on the polarization tensor $M_{j}$ defined by $(2.10)$ (see $[4,17]$ for its properties) and $\breve{\Lambda}_{\alpha}(x)$ represents the inverse Fourier transform of $\Lambda_{\alpha}(\eta)$. 
The method of reconstruction we propose here consists, as in [5], of sampling values of $\Lambda_{\alpha}(\eta)$ at some discrete set of points and then calculating the corresponding discrete inverse Fourier transform. After a rescaling by $-\frac{1}{2}$, the support of this discrete inverse Fourier transform yields the location of the small imperfections $B_{\alpha}$. In other terms, once $\Lambda_{\alpha}(\eta)$ is computed from dynamic boundary measurements on $\Gamma_{c}$, we calculate its inverse Fourier transform. Then the asymptotic formula (2.1) in Theorem 2.1 asserts that this inverse Fourier transform is a distribution supported at the locations $\left\{z_{j}\right\}_{j=1}^{m}$.

Once the locations are known, we may calculate the polarization tensors $\left(M_{j}\right)_{j=1}^{m}$ by solving an appropriate linear system arising from (2.1). These polarization tensors give ideas on the orientation and relative size of the imperfections. From the leading term of $\Lambda_{\alpha}(\eta)$ given by (2.1), we cannot reconstruct more details of the shapes of the domains $B_{j}$. Higher order terms in the asymptotic expansion of $\Lambda_{\alpha}(\eta)$, with respect to $\alpha$, are needed to reconstruct the domains $B_{j}$ with high resolution. The properties of the polarization tensors are described in $[4,17]$.

The theoretical number of data (sampling) points needed for an accurate discrete Fourier inversion of $\Lambda_{\alpha}(\eta)$ follows from Shannon's sampling theorem. We need (conservatively) order $(h / \delta)^{2}$ sampled values of $\Lambda_{\alpha}(\eta)$ to reconstruct, with resolution $\delta$, a collection of imperfections that lie inside a square of side $h$. Numerical experiments in [5] for the two-dimensional (time-independent) inverse conductivity problem confirm this sampling and seem to suggest that the method is quite stable with respect to noise in measurements and errors in the different approximations.

\section{ThE HUM APPROACH TO EXACT BOUNDARY CONTROLLABILITY}

In this section we recall very briefly the numerical method that will be employed in order to solve the exact controllability problem. Full details of the method may be found in [11,19,24-26].

\subsection{Formulation of the problem}

We consider the wave equation with control on a part of the boundary,

$$
\begin{cases}\left(\partial_{t}^{2}-\Delta\right) u=0 & \text { in } Q=\Omega \times(0, T), \\ \left.u\right|_{t=0}=u^{0},\left.\partial_{t} u\right|_{t=0}=u^{1} & \text { in } \Omega, \\ u= \begin{cases}g & \text { on } \Sigma_{c}=\Gamma_{c} \times(0, T), \\ 0 & \text { on } \Sigma \backslash \Sigma_{c}=\Gamma \backslash \Gamma_{c} \times(0, T),\end{cases} \end{cases}
$$

where $\Omega$ is a bounded domain of $\mathbb{R}^{d}, d=2,3$, with boundary $\Gamma$, and $\Gamma_{c} \subset \Gamma$ is the part of the boundary where the control is applied.

The problem of the exact boundary controllability is then: "Given $T, u^{0}, u^{1}$, can we find a control $g$ on $\Sigma_{c}$ such that the solution of (3.1) satisfies

$$
u(x, T)=\partial_{t} u(x, T)=0 \text { in } \Omega ? "
$$

The answer is positive if one takes $T$ sufficiently large and one controls on a set large enough to encounter every ray of geometric optics (see $[11,15]$ ). A systematic and constructive method for computing such a control, $g$, is provided by the Hilbert uniqueness method (HUM) introduced by Lions [30].

\subsection{Description of the HUM}

We describe briefly the HUM for the control of the wave equation (3.1) from a part of the boundary (see [30]). Let

and define the operator

$$
E=H_{0}^{1}(\Omega) \times L^{2}(\Omega), E^{\prime}=H^{-1}(\Omega) \times L^{2}(\Omega)
$$

$$
\Lambda: E \longrightarrow E^{\prime}
$$


as follows:

(1) Take $e=\left\{e^{0}, e^{1}\right\} \in E$ and solve from $t=0$ to $t=T$

$$
\begin{cases}\left(\partial_{t}^{2}-\Delta\right) \varphi=0 & \text { in } Q=\Omega \times(0, T) \\ \left.\varphi\right|_{t=0}=e^{0},\left.\partial_{t} \varphi\right|_{t=0}=e^{1} & \text { in } \Omega \\ \varphi(x, t)=0 & \text { on } \Sigma=\Gamma \times(0, T)\end{cases}
$$

(2) Then solve (backwards) from $t=T$ to $t=0$

$$
\begin{cases}\left(\partial_{t}^{2}-\Delta\right) \psi=0 & \text { in } Q=\Omega \times(0, T), \\ \psi(x, T)=0, & \text { in } \Omega, \\ \psi= \begin{cases}\frac{\partial \varphi}{\partial n} & \text { on } \Sigma_{c}=\Gamma_{c} \times(0, T), \\ 0 & \text { on } \Sigma \backslash \Sigma_{c}=\Gamma \backslash \Gamma_{c} \times(0, T) .\end{cases} \end{cases}
$$

(3) Finally, define the operator $\Upsilon$ as

$$
\Upsilon e=\left\{\psi_{t}(x, 0),-\psi(x, 0)\right\} \in E^{\prime}
$$

We have the original theorem of Lions [30]:

Theorem 3.1 (Lions). Operator $\Upsilon$ is linear and continuous from $E$ onto $E^{\prime}$; moreover, if $T$ is sufficiently large $\left(>T_{\min }=2\left\|x-x_{0}\right\|_{L^{\infty}(\Omega)}\right)$ and if $\Gamma_{c}$ is of the type

$$
\Gamma\left(x_{0}\right)=\left\{x \mid x \in \Gamma,\left(x-x_{0}\right) \cdot n_{x}>0\right\}
$$

where $x_{0} \in \mathbb{R}^{d}$ is an arbitrary point and $n_{x}$ is the outward normal to $\Gamma$ at $x$, then $\Upsilon$ is an isomorphism from $E$ onto $E^{\prime}$.

\subsection{Application of the HUM to the wave equation}

We now apply Theorem 3.1 to the control of the wave equation (3.1). Suppose that

$$
u^{0} \in L^{2}(\Omega), u^{1} \in H^{-1}(\Omega)
$$

are given. Then,

(1) take $f=\left\{u^{1},-u^{0}\right\}-$ i.e. we identify $u$ with $\psi$;

(2) solve $\Upsilon e=f$ to obtain $e^{0}, e^{1}$, the initial data for the $\varphi$ wave equation (3.1);

(3) solve the $\varphi$ wave equation (3.1) forwards in time using $e^{0}, e^{1}$ as initial data;

(4) calculate the normal derivative of the solution $\varphi$ and set $g=\left.\frac{\partial \varphi}{\partial n}\right|_{\Sigma_{0}}$;

(5) solve the $\psi$ wave equation (3.3) backwards in time using $g$ as the boundary data;

(6) finally, set $u=\psi$, then since $\psi(x, T)=0, \psi_{t}(x, T)=0$ was imposed, $g$ (the boundary control) gives the exact boundary controllability with

$$
u(x, T)=\partial_{t} u(x, T)=0, \forall x \in \Omega .
$$

We remark that the operator $\Upsilon$ is symmetric and E-elliptic. These properties imply that, in step (2), $e=f$ can be solved by a conjugate gradient algorithm. We will describe the numerical approximation of the control of system (3.1) in Section 4.3. 


\section{NumericAl DisCRETIZATION OF DYNAMiC LOCALIZATION}

In this section, we present the numerical discretization of our identification problem. First of all, we give the steps of the reconstruction algorithm. Secondly, we briefly describe the finite element method and the time-stepping scheme used for solving the different wave equations contained in this detection problem. Then, we discuss the numerical discretization of the BiGrid HUM method, seen in Section 3. Finally, we present the implementation of the Fourier inversion method and apply it to some tests on the asymptotic formula (2.1).

\subsection{Summary of the identification procedure algorithm}

Let us give the steps of the procedure used by our identification algorithm. First, we suppose a finite number of imperfections, $z_{j}+\alpha B_{j}$ for $j=1, \ldots, m$, with conductivities $\gamma_{j}$. Then, for each $\eta$ in a discrete set $D$ of values,

(1) Compute the solution $u_{\alpha}$ of the wave equation (2.2) by a finite element method to simulate the boundary data $\frac{\partial u_{\alpha}}{\partial n}$ on $\Gamma_{c} \times(0, T)$ for the inverse problem.

(2) Compute the quantity $\frac{\partial u}{\partial n}$ on $\Gamma_{c} \times(0, T)$ which is explicitly known from (2.5).

(3) Calculate the control $g_{\eta}$ of (2.6) via the BiGrid HUM method.

(4) Form the quantity $\Lambda_{\alpha}(\eta)$ from the left hand side of (2.11) with a suitable quadrature formula.

Finally, apply the inverse Fourier transform to $\Lambda_{\alpha}(\eta)$ ( $\eta$ in the Fourier space $D$ ) to compute $\breve{\Lambda}_{\alpha}(x)$ (x in the physical space $\Omega$ ). This leads to the solution of the numerical inverse problem, namely the localization of the centers of the imperfections.

\subsection{Time-space discretization for the wave equation}

In this subsection, we present the numerical resolution of the wave equation: we combine finite elements for the space discretization (see [21]) and finite differences for the time discretization. This will then be used for solving the control problem (2.6) for $g_{\eta}$ and the inhomogeneous wave equation (2.2) for $u_{\alpha}$.

We consider the following, general wave equation with a Dirichlet boundary condition, defined on $\Omega \times(0, T)$ :

$$
\begin{cases}\frac{\partial^{2} u}{\partial t^{2}}-\nabla \cdot(\gamma \nabla u)=f & \text { in } \Omega \times(0, T), \\ u=g & \text { on } \partial \Omega \times(0, T), \\ \left.u\right|_{t=0}=u^{0} & \text { in } \Omega, \\ \left.\frac{\partial u}{\partial t}\right|_{t=0}=u^{1} & \text { in } \Omega,\end{cases}
$$

with $f \in L^{2}\left(L^{2}(\Omega) ;(0, T)\right), g \in H_{0}^{1}(\partial \Omega), \Omega \subset \mathbb{R}^{d}, d=2,3$. We assume that $\gamma \in L^{\infty}(\Omega), \gamma(x)>0, \forall x \in \Omega$, and that $u^{0}, u^{1} \in H^{1}(\Omega)$. Besides, we consider the function $g$ as differentiable in time and satisfying the necessary compatibility conditions with $u^{0}$ and $u^{1}$. In practice, in order to work in the homogeneous space $H_{0}^{1}(\Omega)$, we use a standard extension technique on the function $g$ (see for instance [31]).

For the finite element mesh, we consider shape-regular meshes, $\mathcal{T}_{h}$, that partition the domain $\Omega$ into a set of disjoint elements $\{K\}$, such that $\bar{\Omega}=\cup_{K \in \mathcal{T}_{h}} \bar{K}$. The elements are rectangles (in 2D) or parallelepipeds (in 3D), aligned with the coordinate axes. The diameter of an element $K$ is denoted by $h_{K}$ and the mesh size $h$ is given by $h=\max _{K \in \mathcal{T}_{h}} h_{K}$. We define the following sub-space of $H_{0}^{1}(\Omega)$ on the mesh,

$$
V_{h}=\left\{v_{h} \in C^{0}(\bar{\Omega}) ; v_{h}=0 \text { on } \partial \Omega \text { and }\left.v_{h}\right|_{K} \in Q_{1}, \forall K \in \mathcal{T}_{h}\right\},
$$

where $Q_{1}$ defines the space of polynomials of degree at most one in each of the $d$ variables, thus spanned by $\{1, x, y, x y\}$ when $d=2$, and by $\{1, x, y, z, x y, x z, y z, x y z\}$ when $d=3$. We denote by $\mathbf{I}$ the set of interior nodes (the nodes not belonging to $\Gamma=\partial \Omega$ ) and by $\mathbf{J}$ the set of boundary nodes. Their cardinalities are $N_{I}$ 
and $N_{J}$ respectively. An element $v_{h}$ of $V_{h}$ can be written as

$$
\forall x \in \Omega, v_{h}(x)=\sum_{i=1}^{N_{I}} v_{h}\left(x_{i}\right) \omega_{i}(x),
$$

where $\omega_{i}$ are the $Q_{1}$ basis functions associated to the interior nodes. Moreover, we have

$$
\partial_{t}^{2} v_{h}(x, t)=\sum_{i \in \mathbf{I}} v_{i}^{\prime \prime}(t) \omega_{i}(x)
$$

with $v_{i}^{\prime \prime}(t):=\partial_{t}^{2} v_{h}\left(x_{i}, t\right), i \in \mathbf{I}$. We denote by $P_{h}$ the $L^{2}$-projection onto $V_{h}$. We obtain the linear, second-order system of ordinary differential equations for the discretization of (4.1) by the finite element method

$$
\left\{\begin{array}{l}
M U_{h}^{\prime \prime}(t)+K U_{h}(t)=B_{h} \\
U_{h}(0)=U_{h}^{0} \text { and } U_{h}^{\prime}(0)=V_{h}^{0} \text { given, }
\end{array}\right.
$$

where $U_{h}^{\prime \prime}(t)=\left(u_{i}^{\prime \prime}(t)\right)_{i \in \mathbf{I}}, U_{h}(t)=\left(u_{h}\left(x_{i}, t\right)\right)_{i \in \mathbf{I}}, U_{h}^{0}=P_{h} u^{0}$ and $V_{h}^{0}=P_{h} u^{1}$. The mass matrix, $M$, has coefficients

the stiffness matrix, $K$,

$$
M_{i j}=\int_{\Omega} \omega_{i}(x) \omega_{j}(x) \mathrm{d} x, \quad 1 \leq i, j \leq N_{I}
$$

$$
K_{i j}=\int_{\Omega} \gamma(x) \nabla \omega_{i}(x) \nabla \omega_{j}(x) \mathrm{d} x, \quad 1 \leq i, j \leq N_{I},
$$

and $B_{h} \in \mathbb{C}^{N_{I}}$, the right hand side vector,

$$
B_{i}=\int_{\Omega} f\left(x_{i}, t\right) \omega_{i}(x) \mathrm{d} x-\sum_{j \in \mathbf{J}} K_{i j} g\left(x_{j}, t\right)-\sum_{j \in \mathbf{J}} M_{i j} \frac{\partial^{2} g}{\partial t^{2}}\left(x_{j}, t\right) .
$$

To discretize (4.2) in time, we use the fully implicit, unconditionally stable, Newmark time-stepping scheme (see $[31,33])$.

\subsection{The BiGrid HUM method}

This part is devoted to the computation of the boundary control $g_{\eta}$ of the wave equation in (2.6). This corresponds to the crucial step (3) of the reconstruction algorithm. It is by now well-known that numerical algorithms for the boundary control for the wave equation (2.6) may diverge when the mesh-size tends to zero. These numerical instabilities are due to the high-frequency spurious oscillations. Among the several remedies proposed in the literature $[18,19,28,34,35]$, we choose to use the preconditioned BiGrid algorithm introduced by Glowinski in [24]. We have faithfully followed [11,26] in order to formulate our algorithm. The main numerical novelty here is the generalization of the BiGrid algorithm to the $3 \mathrm{D}$ case. Theoretical results on the convergence of a BiGrid algorithm for the control of the wave equation are very recent. We can cite the article of Ignat and Zuazua [27] that proves the convergence in the case of a finite-difference space semi-discretizations of the $2 \mathrm{D}$ wave equation in the square. The Fourier analysis techniques that they have used can be adapted to any space dimension and to full approximation schemes (finite differences or structured meshes of finite elements), and hence to our problem. At the moment, we observe such behaviour numerically. Our BiGrid algorithm is stable with respect to the mesh-size for regular meshes and smooth data. As an illustration, we report some convergence errors (discrete norms) in Tables 1 and 2 relative to the control of the wave equation on the unit cube using as initial data:

- Table 1: $u^{0}(x, y, z)=10 \sin (\pi x) \sin (\pi y) \sin (\pi z), u^{1}(x, y, z)=0$,

- Table 2: $u^{0}(x, y, z)=\mathrm{e}^{-64\left[(x-0.5)^{2}+(y-0.5)^{2}+(z-0.5)^{2}\right]}, u^{1}(x, y, z)=0$. 
TABLE 1. Oscillatory data on the unit cube with a partial control.

\begin{tabular}{|l|c|c|c|c|}
\hline Mesh size & $h=\frac{1}{8}$ & $h=\frac{1}{16}$ & $h=\frac{1}{32}$ & $h=\frac{1}{64}$ \\
\hline Iterations & 7 & 7 & 6 & 5 \\
\hline$\frac{\left\|u^{0}-u_{h}^{0}\right\|_{L^{2}(\Omega)}}{\left\|u^{0}\right\|_{L^{2}(\Omega)}}$ & 0.0238 & 0.0207 & 0.0103 & 0.0051 \\
\hline$\frac{\left\|u_{h}(T)\right\|_{L^{2}(\Omega)}}{\left\|u_{h}(0)\right\|_{L^{2}(\Omega)}}$ & 0.0467 & 0.0214 & 0.0135 & 0.0087 \\
\hline
\end{tabular}

TABle 2. Smooth exponential initial data on the unit cube with a partial control.

\begin{tabular}{|l|l|l|l|l|}
\hline Mesh size & $h=\frac{1}{8}$ & $h=\frac{1}{16}$ & $h=\frac{1}{32}$ & $h=\frac{1}{64}$ \\
\hline$\frac{\left\|u^{0}-u_{h}^{0}\right\|_{L^{2}(\Omega)}}{\left\|u^{0}\right\|_{L^{2}(\Omega)}}$ & 1.093 & 0.0632 & 0.0328 & 0.0109 \\
\hline$\frac{\left\|u_{h}(T)\right\|_{L^{2}(\Omega)}}{\left\|u_{h}(0)\right\|_{L^{2}(\Omega)}}$ & 0.8060 & 0.1120 & 0.0645 & 0.0353 \\
\hline
\end{tabular}

A partial control on $\Gamma_{c}=\{0 \leq x \leq 1, y=0,0 \leq z \leq 1\} \cup\{x=0,0 \leq y \leq 1,0 \leq z \leq 1\} \cup\{x=1$, $0 \leq y \leq 1,0 \leq z \leq 1\}$ is applied. In our calculations, we fix the time step $\Delta t=h / \sqrt{3}, T=4$ and $\epsilon=10^{-6}$ (see [11] for details on the stopping criterion). We observe a linear in $h$ decay of the $L^{2}$-error as expected from our $Q_{1}$ finite-element approximation.

\subsection{Fourier method}

The localization of the centers, $z_{j}, j=1, \ldots, m$, requires that we numerically compute the inverse Fourier transform of the boundary integral, $\Lambda_{\alpha}(\eta)$ - see Section 2.2. A theoretical framework for the number of points needed is provided by Shannon's Theorem.

Theorem 4.1 (Shannon). If the Fourier transform of a function $h(t)$ is zero for all frequencies greater than a critical frequency, $f_{c}$ (the Nyquist frequency), then the continuous function $h(t)$ can be uniquely determined from a knowledge of its sampled values by the formula

$$
h(t)=T \sum_{n=-\infty}^{\infty} h(n T) \frac{\sin 2 \pi f_{c}(t-n T)}{\pi(t-n T)}
$$

where $T=1 /\left(2 f_{c}\right)$ and $(1 / T)$ is known as the Nyquist sampling rate.

From this theorem we conclude the two following facts:

(1) If the imperfections are known to be contained within a square/cube of side length $2 M$, we must sample $\Lambda_{\alpha}(\eta)$ with a step size $\Delta \eta=1 /(2 M)$.

(2) If we sample in a domain $|\eta|<\eta_{\max }$, then the resolution will be at most $\delta=1 /\left(2 \eta_{\max }\right)$.

Thus we require $N_{\delta}=(2 M / \delta)^{d}, d=2,3$, sampled values of $\Lambda_{\alpha}(\eta)$ in order to reconstruct, at a resolution $\delta$, a set of imperfections contained inside the square/cube of size $2 M$. This implies that we need $\left(4 M \eta_{\max }\right)^{d}$ points.

We now, following the analysis of [32], take a closer look at the quantity $\Lambda_{\alpha}(\eta)$. For clarity, let us assume that $d=2$ and let us examine a simplified form by assuming that our data is of the form

$$
\Lambda(\eta)=\mathrm{e}^{2 \mathrm{i} z \cdot \eta}
$$


for a single imperfection, $z=\left(z_{1}, z_{2}\right)$. Now we sample $\eta$ in the domain $\left[-\eta_{\max }, \eta_{\max }\right] \times\left[-\eta_{\max }, \eta_{\max }\right]$, uniformly with $n^{2}$ points and define the discretization step-size as

$$
\Delta \eta=\frac{2 \eta_{\max }}{n+1}
$$

We obtain the discrete approximation,

$$
\Lambda_{h}(\eta)=\mathrm{e}^{2 \mathrm{i} z_{1}\left(-\eta_{\max }+(k-1) \Delta \eta\right)+2 i z_{2}\left(-\eta_{\max }+(l-1) \Delta \eta\right)}, \quad 1 \leq k, l \leq n .
$$

Applying the inverse discrete Fourier transform (IDFT) to this quantity, we obtain

$$
\frac{1}{n^{2}} \sum_{k=1}^{n} \sum_{l=1}^{n} \mathrm{e}^{2 \mathrm{i} z_{1}\left(-\eta_{\max }+(k-1) \Delta \eta\right)+2 i z_{2}\left(-\eta_{\max }+(l-1) \Delta \eta\right)} \mathrm{e}^{2 \pi i\left(\frac{(k-1)}{n}(r-1)\right)+2 \pi i\left(\frac{(l-1)}{n}(s-1)\right)}
$$

for all $1 \leq r, s \leq n$. Let us look at the first coordinate,

$$
\check{\Lambda}_{1}=\frac{1}{n} \sum_{k=1}^{n} \mathrm{e}^{2 \mathrm{i} z_{1}\left(-\eta_{\max }+(k-1) \Delta \eta\right)} \mathrm{e}^{2 \pi i\left(\frac{(k-1)}{n}(r-1)\right)} .
$$

An elementary calculation yields

$$
\left|\check{\Lambda}_{1}\right|=\left|\frac{1}{n} \frac{\sin \left(2 \eta_{\max } z_{1}\right)}{\sin \left[\pi\left(\frac{z_{1} \Delta \eta}{\pi}+\frac{r-1}{n}\right)\right]}\right|
$$

For $n$ large $(n \rightarrow \infty),\left|\check{\Lambda}_{1}\right| \rightarrow 0$ unless the quantity in the denominator equals zero. This will occur when $\sin k \pi=0, k=0, \pm 1, \ldots$ which means that $\left(\frac{z_{1} \Delta \eta}{\pi}+\frac{r-1}{n}\right)$ must be close to an integer. So, once the sign of $z_{1}$ is fixed, in order to approach only one integer value (and thus observe a single peak in the Fourier transform), the following condition must hold:

$$
\left|\frac{z_{1} \Delta \eta}{\pi}\right| \leq \frac{1}{2}
$$

since $0 \leq \frac{r-1}{n} \leq 1$

For large values of $\eta_{\max }$, we begin to observe instabilities due to the exponential terms in the asymptotic formula that become highly oscillatory. In order to control these oscillations, we impose the truncation $\|\eta\| \leq$ $\left\|\left(\eta_{*}, \eta_{*}\right)^{T}\right\|$ in a discrete 2-norm and set the quantity $\Lambda_{\alpha}(\eta)=0$ for all other values of $\eta$ as was done in [12,32].

\subsubsection{Test based on the asymptotic formula (2.1)}

We recall the asymptotic formula (2.1), neglecting the higher order term,

$$
\Lambda_{\alpha}(\eta)=\alpha^{d} \sum_{j=1}^{m}\left(\frac{\gamma_{0}}{\gamma_{j}}-1\right) \mathrm{e}^{2 \mathrm{i} \eta \cdot z_{j}}\left[M_{j}(\eta) \cdot \eta-|\eta|^{2}\left|B_{j}\right|\right], \quad d=2,3
$$

Let us illustrate through a $3-\mathrm{D}$ case the inherent precision and robustness of this formula. We denote by $N_{e}$ the number of Fourier points for each component. We report in Figure 2 the successful localization of three imperfections via the Shannon sampling approach without truncation by taking $N_{e}=128, \eta_{\max }=64$. Extensive tests of the formula with different sampling strategies can be found in [22]. 

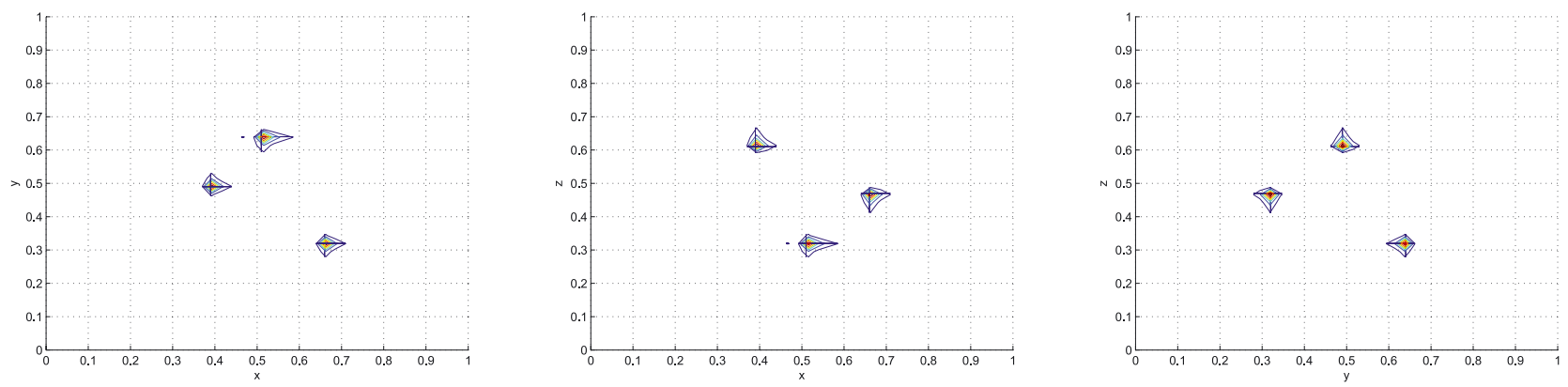

FIGURE 2. Shannon sampling approach without truncation, using the asymptotic formula, $z_{1}=(0.51,0.64,0.32), z_{2}=(0.66,0.32,0.47)$ and $z_{3}=(0.39,0.49,0.61)$.

TABLE 3. Discretization parameters for all numerical simulations.

\begin{tabular}{|c|c|c|}
\hline Parameter & $2-\mathrm{D}$ & 3 -D \\
\hline \hline$N$ & 16,32 & 14 \\
\hline$h$ & $1 / N$ & $1 / N$ \\
\hline$\Delta t$ & $h / \sqrt{2}$ & $h / \sqrt{3}$ \\
\hline$T$ & $3>2 \sqrt{2}$ & $4>2 \sqrt{3}$ \\
\hline$\epsilon$ & $10^{-6}$ & $10^{-6}$ \\
\hline
\end{tabular}

\section{Numerical Simulations}

We present here some numerical results of our identification algorithm. In all examples, we take the domain $\Omega$ to be the unit square $[0,1]^{2}$ or the unit cube $[0,1]^{3}$. We use $Q_{1}$ finite elements for the space discretization and an implicit, unconditionally stable Newmark scheme for the time discretization (see Sect. 4.2). We also suppose that when the control is active on the entire boundary we take a final time, $T$, according to Table 3 . The spatial discretization, $h$, and the corresponding time step $\Delta t$ are also given in Table 3 , as well as the stopping criterion of the BiGrid HUM method, $\epsilon$. The cutoff function, $\beta$, is equal to 1 on a subdomain $\Omega^{\prime}=(0.2,0.8)^{d}, d=2,3$. The conductivity $\gamma_{0}$ of the background medium is equal to 1 . The Fourier parameters depend on the geometry considered (number of imperfections and location of each imperfection) and the resolution required. In all the figures, we plot contours of the inverse Fourier transform, $\breve{\Lambda}_{\alpha}(x)$.

\subsection{Numerical localization of imperfections in $2 \mathrm{D}$}

We now present results of the numerical localization procedure, following the steps laid out in Section 4.1.

\subsubsection{The Shannon sampling approach with truncation}

In Figure 3 we show results for localization using the Shannon approach with truncation of the $\eta$-values. In both cases, we fix $\alpha=0.04, N_{e}=128$ and $\eta_{\max }=33$. The truncation is quite severe, $\eta_{*}=11$, but we obtain satisfactory results. We have also applied various windowing methods for the computation of the inverse Fourier transform. These simulations can be found in the thesis [22].

\subsubsection{Limited-view data}

Since the HUM method permits us to control on a part of the boundary, we can simulate cases where numerical data are available only on a subset of $\partial \Omega$. In this case, the causality of the wave equation will imply the need of a longer control time $T$ and the eventuality of an increase in the number of iterations required for the HUM algorithm to converge. A detailed presentation can be found in [11]. In Figure 4 we report 


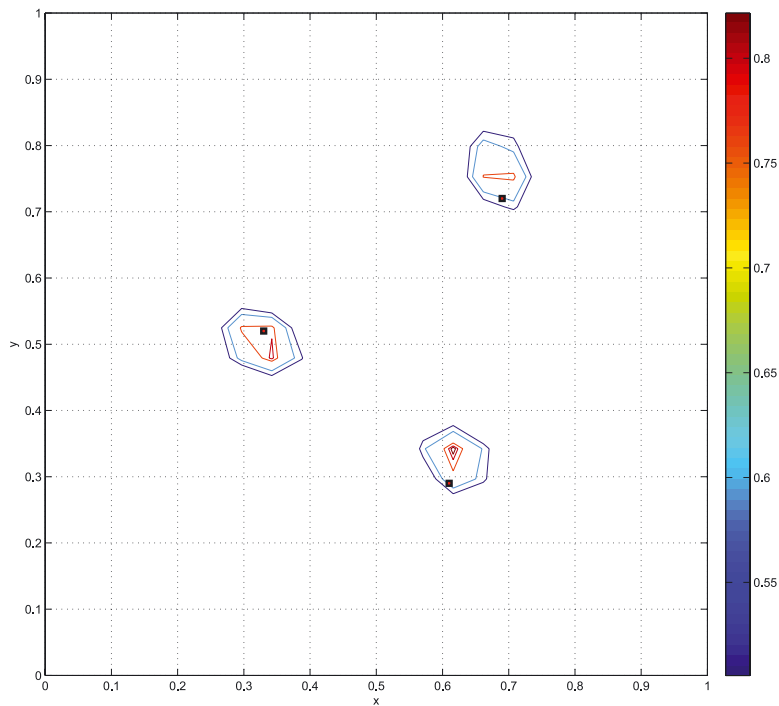

(a) $z_{1}=(0.33,0.52), z_{2}=(0.61,0.29), z_{3}=(0.69,0.72)$

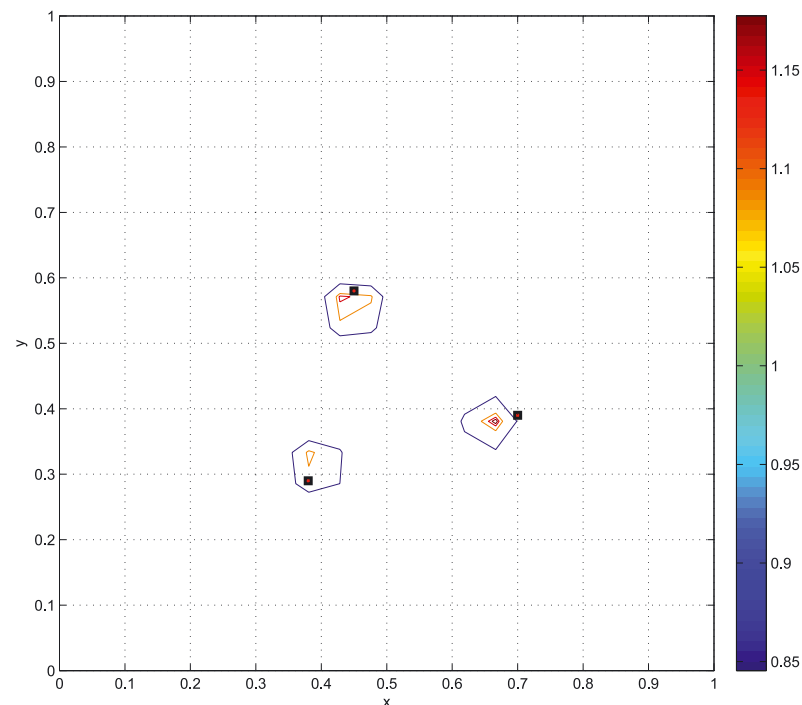

(b) $z_{1}=(0.45,0.58), z_{2}=(0.38,0.29), z_{3}=(0.70,0.39)$

FiguRE 3. Localization in $2 \mathrm{D}$ of three imperfections, with thresholding at $\|\eta\| \leq\|(11,11)\|_{l^{2}}$. The true centers are marked by the small squares.

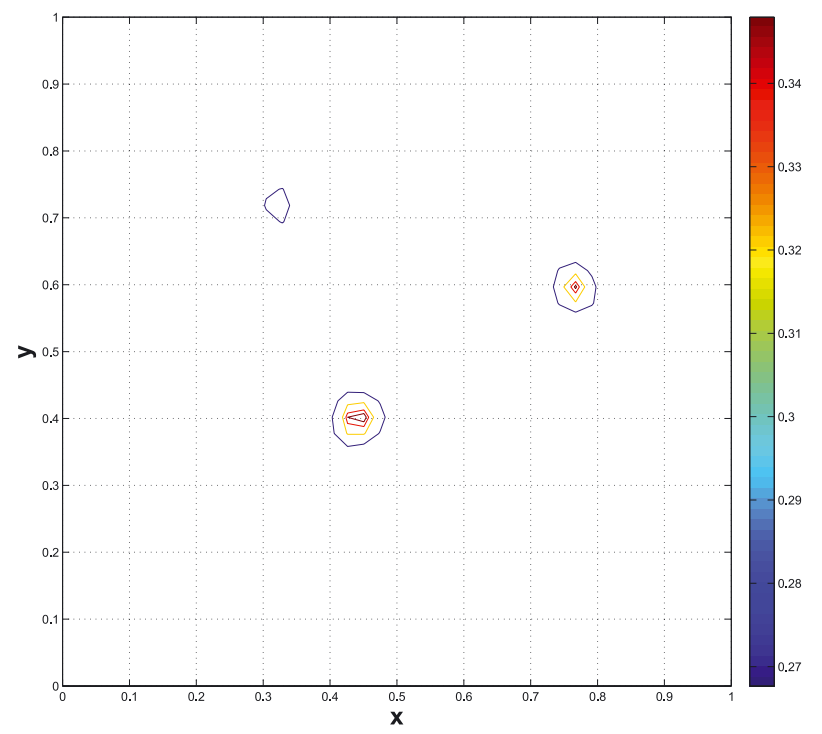

FiguRE 4. Localization in 2D with limited-view data.

the result of a computation with three imperfections, $z_{1}=(0.43,0.39), z_{2}=(0.32,0.67), z_{3}=(0.73,0.58)$. The control boundary is $\{x=1,0 \leq y \leq 1\} \cup\{0 \leq x \leq 1, y=0\}$, the control time $T=3$, the conductivity is $\gamma_{j}=10, j=1,2,3$ and $h=1 / 16$. The parameters of the Fourier discretization are: $N_{e}=128, \eta_{\max }=128$ and $\eta_{*}=12$.

This result is very important, since it illustrates the potential of our algorithm for treating more realistic cases, where full measurements are rarely available. 

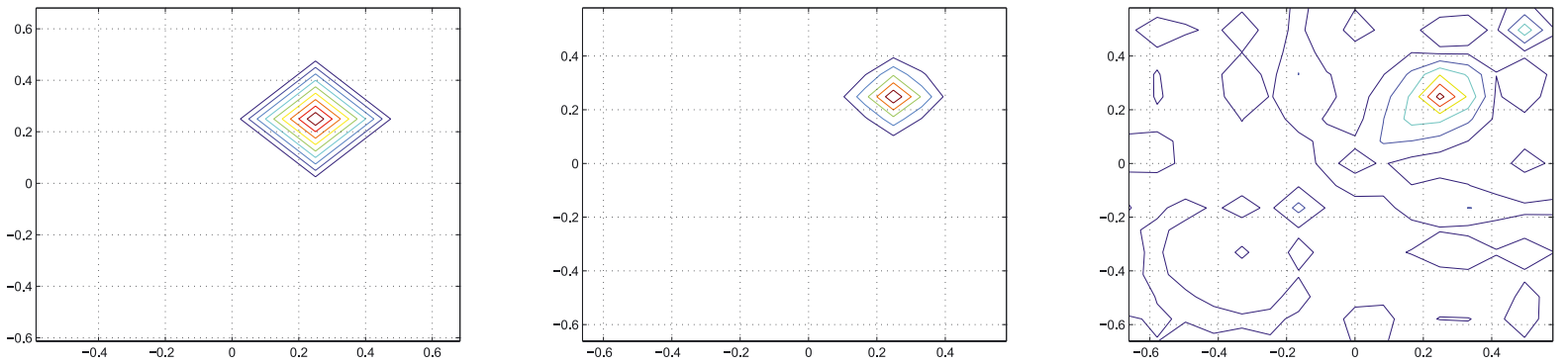

FIGURE 5. Localization in $3 \mathrm{D}$ of a single imperfection at $z=(0.25,0.25,0.25)$ : asymptotic formula (left), asymptotic formula with truncation (center), numerical localization (right) views of $x-y$ plane at $z=0.25$.
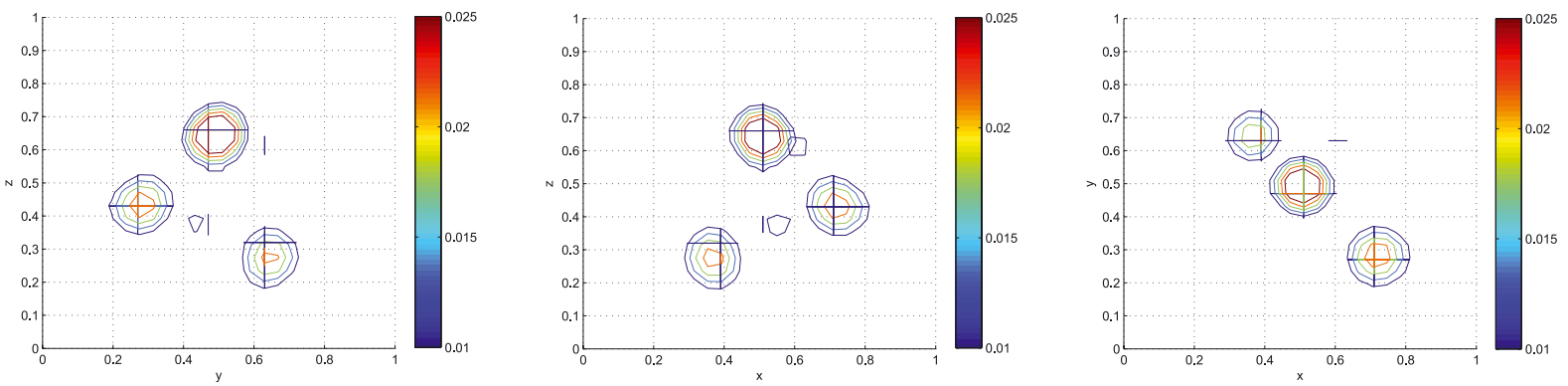

Figure 6. Localization of three imperfections, centered at $z_{1}=(0.39,0.63,0.32), z_{2}=$ $(0.71,0.27,0.43), z_{3}=(0.51,0.47,0.66)$. Views from the $x$-, $y$ - and $z$-directions.

\subsection{Numerical localization of imperfections in 3D}

We now turn to the three-dimensional case. We consider the Shannon sampling approach and assume that we have either full or partial numerical boundary data.

\subsubsection{The Shannon sampling approach with truncation}

In the first example, we have taken one imperfection with equal abscissa and ordinates. The imperfection is situated at $(0.25,0.25,0.25)$ with a radius $\alpha=0.05$. The conductivity of the medium is equal to 1 and that of the imperfection is equal to 10. The number of data (sampling) points for the calculation of $\Lambda_{\alpha}$ is $N_{e} \times N_{e} \times N_{e}=16 \times 16 \times 16$ and the space step $h=1 / 12$. The Fourier inversion is performed with $\eta_{\max }=19$ and a truncation at $\eta_{*}=9$. In Figure 5 we compare the numerical result with that obtained from the asymptotic formula, with and without truncation.

The second test case has three imperfections, centered at $z_{1}=(0.39,0.63,0.32), z_{2}=(0.71,0.27,0.43)$, $z_{3}=(0.51,0.47,0.66)$. The number of data (sampling) points for the calculation of $\Lambda_{\alpha}$ is $N_{e} \times N_{e} \times N_{e}=$ $64 \times 64 \times 64$, with $\eta_{\max }=40$ and a truncation at $\eta_{*}=9$. The spatial discretization step is $h=1 / 14$, the radius of the imperfections $\alpha=0.02$ and the conductivity $\gamma_{j}=10$. In Figure 6 we observe that all three imperfections are well located. A 3D view of the localized imperfections is shown in Figure 7.

Finally, we confirm the robustness of our method by a test with four imperfections centered at $z_{1}=$ $(0.66,0.32,0.47), z_{2}=(0.55,0.71,0.39), z_{3}=(0.39,0.63,0.31), z_{4}=(0.71,0.42,0.74)$. We fix the parameters $N_{e}=64, \eta_{\max }=40$ and $\eta_{*}=9$. The radius of the imperfections is now $\alpha=0.01$ and the conductivity $\gamma_{j}=10$. In Figures 8 and 9 , we can observe that all four imperfections are well located. 


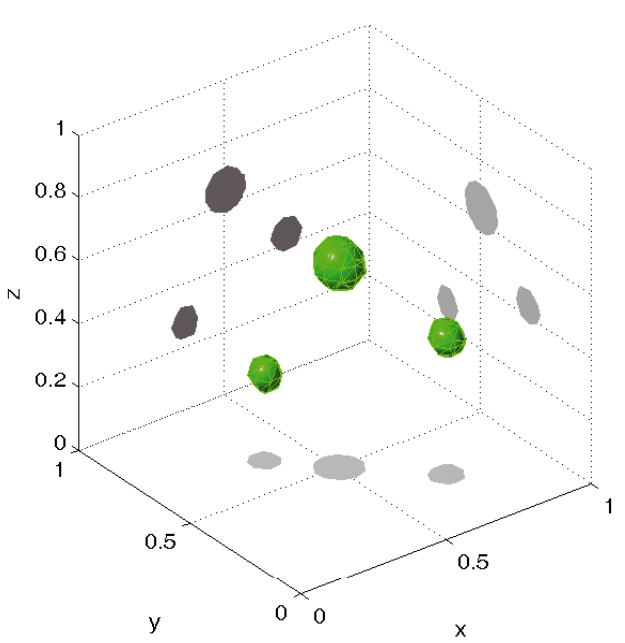

FiguRE 7. Localization of three imperfections centered at $z_{1}=(0.39,0.63,0.32), z_{2}=$ $(0.71,0.27,0.43), z_{3}=(0.51,0.47,0.66) .3 \mathrm{D}$ view.
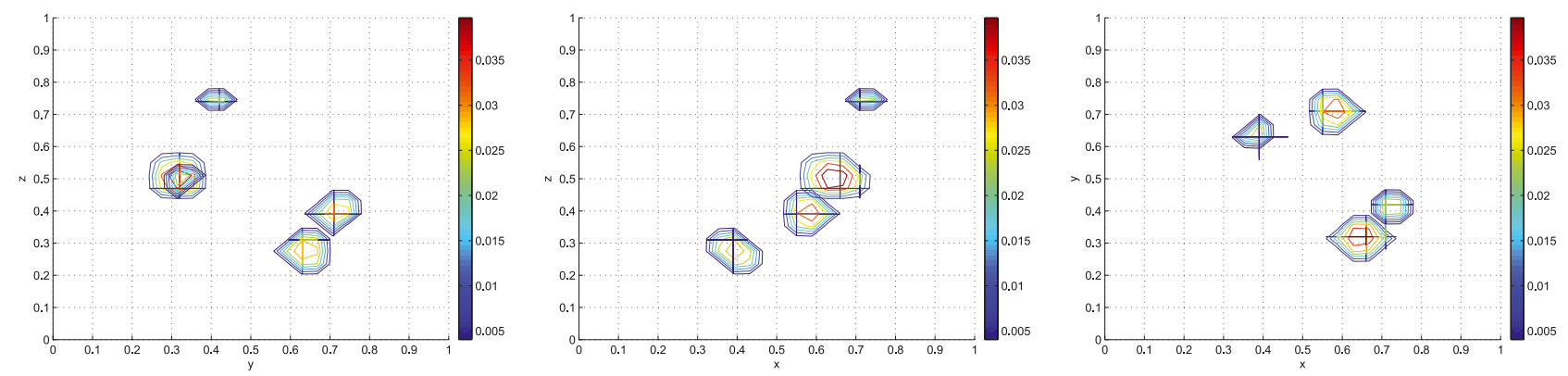

FiguRE 8. Localization in 3D of four imperfections, centered at $z_{1}=(0.66,0.32,0.47), z_{2}=$ $(0.55,0.71,0.39), z_{3}=(0.39,0.63,0.31), z_{4}=(0.71,0.42,0.74)$. Views from the $x-, y$ - and $z$-directions.

\subsubsection{Limited-view data}

We consider again the second test of the previous section, using now partial measurements on $\Gamma_{c}=\{0 \leq$ $x \leq 1, y=0,0 \leq z \leq 1\} \cup\{x=0,0 \leq y \leq 1,0 \leq z \leq 1\} \cup\{x=1,0 \leq y \leq 1,0 \leq z \leq 1\}$. In other words, measurements are assumed to be available on only three adjacent faces of the cube. In Figure 10 we notice that the imperfections are still well located. Relative errors for the localization of the centers are given in Table 4. These results illustrate both the accuracy and the robustness of the method. Note that the theoretical resolution is $\delta=1 / 2 \eta_{\max }=0.0125$.

\subsection{Some remarks on convergence}

To end this section, we discuss various aspects related to the convergence of our numerical identification algorithm. To fix ideas, we took a single imperfection, centered at $z=[0.5,0.5]$, in the domain $[0,1] \times[0,1]$. We considered two meshes, $20 \times 20$ with $h=0.05$ and $40 \times 40$ with $h=0.025$. For each mesh we ran the detection algorithm for imperfections of radius $\alpha=0.1,0.05,0.025,0.0125$. We define the error as $e=\left\|z-z_{c}\right\| /\|z\|$, where $z_{c}$ is the computed center. 


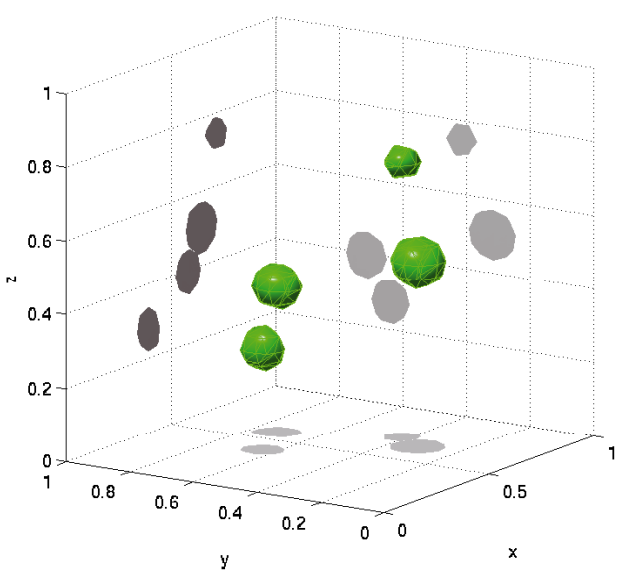

Figure 9. Localization of four imperfections centered at $z_{1}=(0.66,0.32,0.47), z_{2}=$ $(0.55,0.71,0.39), z_{3}=(0.39,0.63,0.31), z_{4}=(0.71,0.42,0.74) .3 \mathrm{D}$ view.
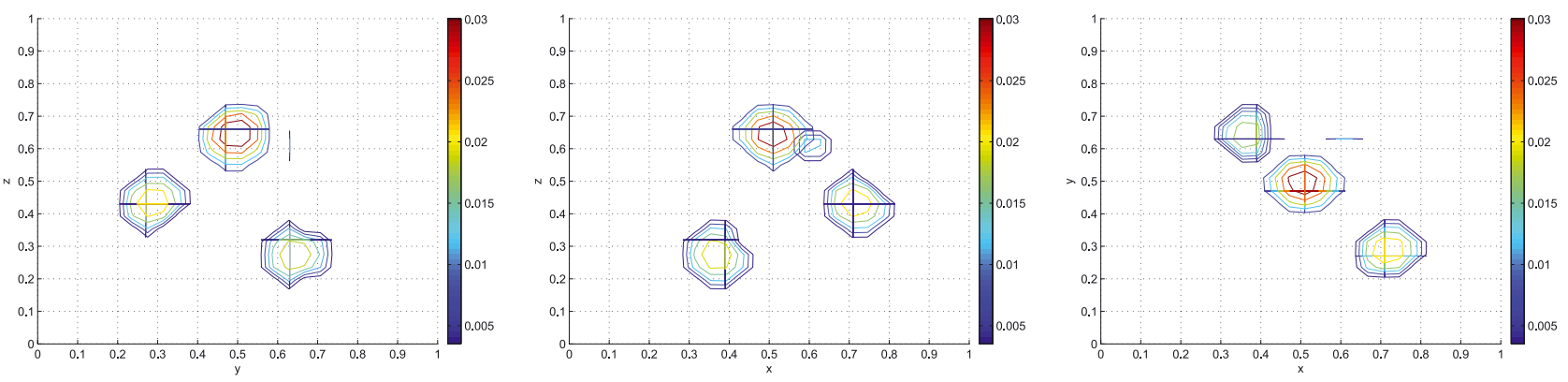

FIGURE 10. Localization in $3 \mathrm{D}$ with limited-view data of three imperfections, centered at $z_{1}=(0.39,0.63,0.32), z_{2}=(0.71,0.27,0.43), z_{3}=(0.51,0.47,0.66)$. Views from the $x-, y$ - and $z$-directions.

TABLE 4. Localization of the centers of three imperfections. Relative localization errors (total control versus partial control).

\begin{tabular}{|l|c|c|}
\hline Theoretical centers & Total control & Partial control \\
\hline$z_{1}=(0.39,0.63,0.32)$ & 0.072 & 0.072 \\
\hline$z_{2}=(0.71,0.27,0.43)$ & 0.007 & 0.007 \\
\hline$z_{3}=(0.51,0.47,0.66)$ & 0.033 & 0.052 \\
\hline
\end{tabular}

We first consider the convergence behaviour of the localization error as a function of the size of the imperfections - see Table 5 (left). Our computations reveal that as long as the mesh size is sufficiently small, we can accurately detect imperfections of any size. We start to lose precision as soon as the imperfection radius is of the order of the mesh size. However, the imperfection center is still accurately recovered. This can be explained by the robustness of the Fourier approach where we can increase the number of points in the Fourier domain and thus reduce the step-size while still maintaining a large enough bandwidth in order to avoid truncation effects. The computations show that the only critical parameters are $\eta_{\max }, N_{e}$ and $\eta_{*}$. In other words, by adjusting these three Fourier parameters, we can obtain an equally good precision, $e$, for the two meshes and for all imperfection radii - see Table 5 (right) and Table 6 . 
TABLE 5. Convergence of the Fourier detection algorithm as a function of the imperfection size (on left) and of $\eta_{\max }$ (on right) for the $20 \times 20$ mesh with $h=0.05$.

\begin{tabular}{|c|c|c|c|c|}
\hline$\alpha$ & $N_{e}$ & $\eta_{\max }$ & $\eta_{*}$ & $e$ \\
\hline \hline 0.1 & 128 & 80 & 6 & 0.0149 \\
\hline 0.05 & 128 & 80 & 6 & 0.0149 \\
\hline 0.025 & 128 & 80 & 6 & 0.0149 \\
\hline
\end{tabular}

\begin{tabular}{|c|c|c|c|c|}
\hline$\alpha$ & $N_{e}$ & $\eta_{\max }$ & $\eta_{*}$ & $e$ \\
\hline \hline 0.1 & 64 & 40 & 6 & 0.0526 \\
\hline 0.1 & 64 & 50 & 6 & 0.0301 \\
\hline 0.1 & 64 & 60 & 6 & 0.0150 \\
\hline
\end{tabular}

TABLE 6. Convergence of the Fourier detection algorithm as a function of the imperfection size for the $40 \times 40$ mesh with $h=0.025$.

\begin{tabular}{|c|c|c|c|c|}
\hline$\alpha$ & $N_{e}$ & $\eta_{\max }$ & $\eta_{*}$ & $e$ \\
\hline \hline 0.1 & 128 & 80 & 6 & 0.0149 \\
\hline 0.05 & 128 & 80 & 6 & 0.0149 \\
\hline 0.025 & 128 & 80 & 6 & 0.0149 \\
\hline 0.0125 & 128 & 80 & 6 & 0.0149 \\
\hline
\end{tabular}

The convergence of our implementation of the HUM algorithm has already been discussed in Section 4.3.

Concerning the convergence of the complete identification algorithm with respect to the (space-time) mesh size, we observe that this is related to a complex interplay between the HUM algorithm, the FFT and the numerical integration used to evaluate the boundary measurements. In particular, we note that numerical FFT precision is highly dependent on the effects of aliasing, truncation and correct sampling in general (see Sect. 4.4 and [16]). Thus we conclude that if the FFT and the numerical integration are supposed accurate enough, the overall convergence will be that of the HUM algorithm i.e. first order in space and time.

Remark 5.1. In order to reduce the CPU time needed by our identification algorithm, we have used Message Passing Interface (MPI) commands for a parallel implementation in Fortran 90. Two levels of parallelism are possible:

- coarse grain, where we exploit the MPI commands for looping on chunks of different values of $\eta$;

- and fine grain, where the linear solvers are parallelized using the PETSc package [14].

In the first case, the speed-up should be linear in the number of processors. In the second case, we expect the speed-up to be superlinear. We present in Figure 11 a graph of CPU time versus the number of processors. For a sequence of $2 \mathrm{D}$ computations, we have taken $h=1 / 32$ and $N_{e}=16$, thus yielding 1024 sample points in $x-y$ space, 135 time steps and 256 sample points in Fourier space.

We remark that we obtain a perfectly linear speed-up in the number of processors.

\section{Conclusion And PERSPECtives}

This numerical study has proven the feasibility and the robustness of the dynamic localization procedure proposed by Ammari in [2] that is based on the wave equation. The two central parts of this procedure are the computation of a control function, via a geometric control method, and a Fourier inversion of a boundary integral. We have carefully implemented these two parts. The first using an algorithm based on the HUM (Hilbert Uniqueness Method) with a BiGrid, finite-element spatial approximation and a Newmark scheme in time, and the second using Shannon's theorem and windowing functions. Our implementation of the HUM also permits the study of limited-view data where the observations are available on only a part of the frontier of the object/domain that we are imaging.

The results obtained, in $2 \mathrm{D}$ and in $3 \mathrm{D}$, are remarkably accurate. We can localize an arbitrary number of randomly placed imperfections with good precision. Particularly encouraging are the good results obtained in the limited-view cases that show that if we can construct the control function accurately enough, we can 


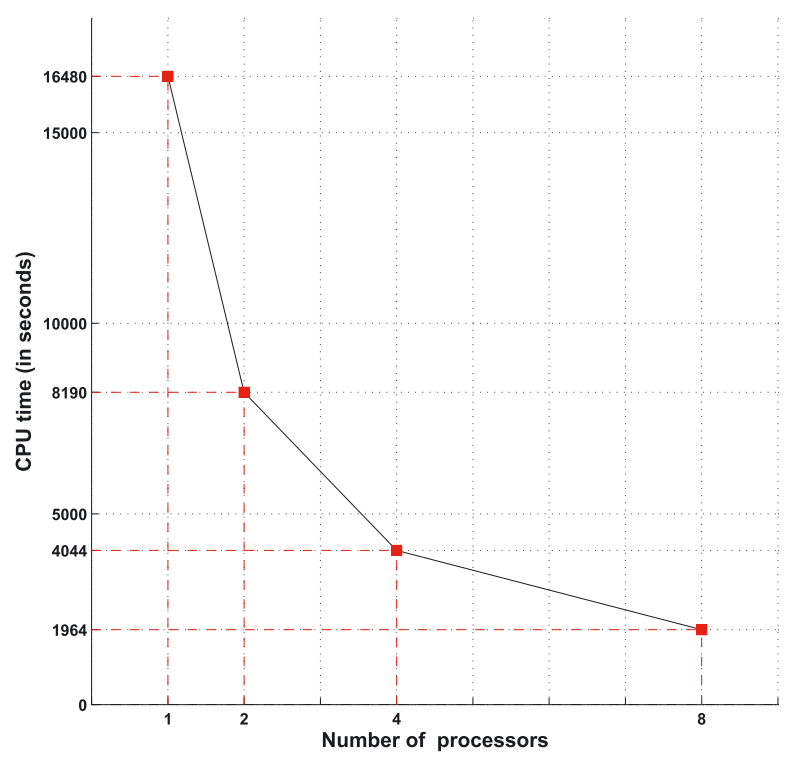

Figure 11. Performance curve for the localization of one imperfection with parallelization.

obtain the same localization precision with limited-view data as that which can be obtained from full boundary data.

The perspectives are numerous. First of all, we intend to extend the treatment to general geometries. However, here we need to ensure the applicability of the BiGrid method which has been based here on a structured mesh. In order to overcome this, we could resort to uniformly controllable approaches as in [13], or spectral approaches [29], that can both be used with unstructured meshes. A second perspective is to treat coupled photoacoustic [9] and magnetoacoustic [8] approaches in the dynamic regime. There is also the possibility of extending the complete localization procedure to Maxwell's equations, for which the theory exists (see [3]) but the numerical implementation of the geometric control still remains to be done.

Acknowledgements. The authors would like to thank the referees whose highly relevant remarks greatly improved the final form of this paper.

\section{REFERENCES}

[1] C. Alves and H. Ammari, Boundary integral formulae for the reconstruction of imperfections of small diameter in an elastic medium. SIAM J. Appl. Math. 62 (2002) 94-106.

[2] H. Ammari, An inverse initial boundary value problem for the wave equation in the presence of imperfections of small volume. SIAM J. Control Optim. 41 (2002) 1194-1211.

[3] H. Ammari, Identification of small amplitude perturbations in the electromagnetic parameters from partial dynamic boundary measurements. J. Math. Anal. Appl. 282 (2003) 479-494.

[4] H. Ammari and H. Kang, Polarization and Moment Tensors: With Applications to Inverse Problems and Effective Medium Theory, Applied Mathematical Sciences 162. Springer-Verlag, New York (2007).

[5] H. Ammari, S. Moskow and M. Vogelius, Boundary integral formulas for the reconstruction of electromagnetic imperfections of small diameter. ESAIM: COCV 62 (2002) 94-106.

[6] H. Ammari, P. Calmon and E. Iakovleva, Direct elastic imaging of a small inclusion. SIAM J. Imaging Sci. 1 (2008) 169-187.

[7] H. Ammari, H. Kang, E. Kim, K. Louati and M. Vogelius, A MUSIC-type algorithm for detecting internal corrosion from electrostatic boundary measurements. Numer. Math. 108 (2008) 501-528.

[8] H. Ammari, Y. Capdeboscq, H. Kang and A. Kozhemyak, Mathematical models and reconstruction methods in magnetoacoustic imaging. Eur. J. Appl. Math. 20 (2009) 303-317. 
[9] H. Ammari, E. Bossy, V. Jugnon and H. Kang, Mathematical Modelling in Photo-Acoustic Imaging. SIAM Rev. (to appear).

[10] H. Ammari, M. Asch, L.G. Bustos, V. Jugnon and H. Kang, Transient wave imaging with limited-view data. SIAM J. Imaging Sci. (submitted) preprint available from http://www.cmap.polytechnique.fr/ ammari/preprints.html.

[11] M. Asch and G. Lebeau, Geometrical aspects of exact boundary controllability for the wave equation - A numerical study. ESAIM: COCV 3 (1998) 163-212.

[12] M. Asch and S.M. Mefire, Numerical localizations of 3D imperfections from an asymptotic formula for perturbations in the electric fields. J. Comput. Math. 26 (2008) 149-195.

[13] M. Asch and A. Münch, Uniformly controllable schemes for the wave equation on the unit square. J. Optim. Theory Appl. 143 (2009) 417-438.

[14] S. Balay, K. Buschelman, W.D. Gropp, D. Kaushik, M.G. Knepley, L. Curfman McInnes, B.F. Smith and H. Zhang, PETSc Web page, http://www.mcs.anl.gov/petsc (2001).

[15] C. Bardos, G. Lebeau and J. Rauch, Sharp sufficient conditions for the observation, control and stabilization of waves from the boundary. SIAM J. Control Optim. 30 (1992) 1024-1065.

[16] E.O. Brigham. The fast Fourier transform and its applications. Prentice Hall, New Jersey (1988).

[17] Y. Capdebosq and M.S. Vogelius, A review of some recent work on impedance imaging for inhomogeneities of low volume fraction, in Contemporary Mathematics 362, C. Conca, R. Manasevich, G. Uhlmann and M.S. Vogelius Eds., AMS (2004) $69-88$.

[18] C. Castro and S. Micu, Boundary controllability of a linear semi-discrete 1-D wave equation derived from a mixed finite element method. Numer. Math. 102 (2006) 413-462.

[19] C. Castro, S. Micu and A. Münch, Numerical approximation of the boundary control for the wave equation with mixed finite elements in a square. IMA J. Num. Anal. 28 (2008) 186-214.

[20] D.J. Cedio-Fengya, S. Moskow and M. Vogelius, Identification of conductivity imperfections of small diameter by boundary measurements. Continuous dependence and computational reconstruction. Inv. Probl. 14 (1998) 553-595.

[21] P.G. Ciarlet, The finite element method for elliptic problems, Studies in Mathematics and Its Applications 4. North-Holland Publishing Company (1978).

[22] J.-B. Duval, Identification dynamique de petites imperfections. Ph.D. Thesis, Université de Picardie Jules Verne, France (2009).

[23] L.C. Evans, Partial Differential Equations, Grad. Stud. Math. 19. AMS, Providence (1998).

[24] R. Glowinski, Ensuring well posedness by analogy; Stokes problem and boundary control for the wave equation. J. Comput. Phys. 103 (1992) 189-221.

[25] R. Glowinski and J.-L. Lions, Exact and approximate controllability for distributed parameter systems. Acta Numer. 4 (1995) 159-328.

[26] R. Glowinski, C.H. Li and J.-L. Lions, A numerical approach to the exact controllability of the wave equation (I). Dirichlet controls: Description of the numerical methods. Jpn. J. Appl. Math. 7 (1990) 1-76.

[27] L.I. Ignat and E. Zuazua, Convergence of a two-grid method algorithm for the control of the wave equation. J. Eur. Math. Soc. 11 (2009) 351-391.

[28] J.A. Infante and E. Zuazua, Boundary observability for the space discretization of the one-dimensional wave equation. ESAIM: M2AN 33 (1999) 407-438.

[29] G. Lebeau and M. Nodet, Experimental study of the HUM control operator for linear waves. Experimental Mathematics 19 (2010) 93-120.

[30] J.-L. Lions, Contrôlabilité exacte, Perturbations et Stabilisation de Systèmes Distribués, Tome 1, Contrôlabilité exacte. Masson, Paris (1988).

[31] A. Quarteroni and A. Valli, Numerical Approximation of Partial Differential Equations. Springer (1997).

[32] M. Vogelius and D. Volkov, Asymptotic formulas for perturbations in the electromagnetic fields due to the presence of inhomogeneities of small diameter. ESAIM: M2AN 34 (2000) 723-748.

[33] W.L. Wood, Practical time-stepping schemes. Oxford Applied Mathematics and Computing Science Series, Clarendon Press, Oxford (1990).

[34] E. Zuazua, Boundary observability for the finite-difference space semi-discretizations of the 2-D wave equation in the square. J. Math. Pures Appl. 78 (1999) 523-563.

[35] E. Zuazua, Propagation, observation and control of waves approximated by finite difference methods. SIAM Rev. 47 (2005) $197-243$. 